\title{
openheart Examining uncertainty in the primary prevention of cardiovascular disease
}

\author{
Rahul Bahl
}

To cite: Bahl R. Examining uncertainty in the primary prevention of cardiovascular disease. Open Heart 2016;3: e000401. doi:10.1136/ openhrt-2016-000401

Accepted 21 January 2016

\section{SLinked}

- http://dx.doi.org/10. 1136/openhrt-2015-000343

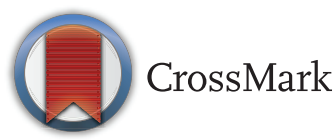

Open Heart, BMJ Journals, BMA House, Tavistock Square, London, WC1H 9JR

Correspondence to Dr Rahul Bahl; rahulbahl2004@hotmail.com
Making decisions in areas of uncertainty is one of the more challenging aspects of free will in general and medicine in particular. This is especially the case when those decisions relate to matters of life and death. Finegold $e t a l^{1}$ present a paper in Open Heart that may considerably complicate a scenario usually thought of as relatively straightforward, namely, the decision to start primary prevention therapy with statins to reduce the risk of death from cardiovascular disease (CVD).

At the heart of the study is a computer model generated from national mortality statistics and a validated cardiovascular risk score. A hypothetical population is generated with known probabilities of cardiovascular and non-cardiovascular death (eg, 1000 50-year-old male non-smokers with normal cholesterol and blood pressure). These probabilities are used to calculate the lifespans of the imagined individuals in this population. The risk of cardiovascular death is then reduced (simulating the effect of statin therapy) and the model run again to see what the effect is on the lifespan of each person. The important thing to realise is that with these models it is possible to examine and re-examine the lifespans of the same set of individuals over and over while altering the variables and observing the effects. Clearly, such experiments are not possible using actual human beings.

There are a number of key findings. First, in any group of individuals with a given risk of CVD, only a few gain any lifespan from reducing their CVD risk, with the vast majority seeing no increase at all. Second, in a group with a higher level of CVD risk, there are greater proportions of people who benefit, with those who do benefit tending to gain a similar amount of lifespan regardless of CVD risk level. Finally, it shows that starting therapy earlier in life gives a greater increase in lifespan than starting it later.

Conclusions such as this have important implications for clinical practice and so should not be accepted unthinkingly. It is important to remember that, even though the paper is based on real UK mortality figures, the findings are the result of hypothetical modifications of these. It is a statistical thought experiment. The biggest flaws in any model such as this lie in the implicit assumptions, and there are a few here that warrant discussion.

First, the model treats the risks of cardiovascular and non-cardiovascular mortality as independent of each other. This is not the case in reality and examples can be given. The authors acknowledge the existence of confounding factors, such as smoking-a factor that certainly increases the risk of death from CVD but also increases the risk of death from chronic obstructive pulmonary disease and cancer. Therefore, a smoker, compared to a non-smoker, might gain more lifespan from starting a statin due to his or her increased CVD risk but, conversely, could gain less due to the increased risk of dying from something else.

The co-existence of multiple disease processes is also a confounder in itself. Official statistics rely largely on data from death certification by doctors where a proximal cause of death is designated in section 1 and other contributing diseases are designated in section 2. The section 1 disease is usually reported in official statistics as the cause of death, ${ }^{2}$ and this is what is used in Finegold et als model to distinguish between CVD and non-CVD risk. However, actual pathophysiology does not proceed in this manner. A patient with ischaemic heart disease who also suffers from multiple chronic pathologies in other organ systems will, unfortunately, have a worse prognosis than the same patient with the same severity of ischaemic heart disease alone. The former patient's demise might be statistically coded as being primarily cardiovascular or non-cardiovascular depending on the circumstances, but the reality is that such a person dies from the accumulated physiological burden of multiple disease processes rather than the linear progression of each pathology individually. To put it another way, 
reducing the risk of dying from one disease will reduce the risk of dying from any disease. It is difficult to know the extent of these interactions in individuals let alone in populations. The effect is that the gain in lifespan from reducing CVD risk is going to be more widespread than that predicted by Finegold $e t a l$ s model.

A large proportion of the population on whom the model's numbers are based will already have been on primary prevention therapy. Data from 2006/2007 suggest around $10 \%$ of the population were then taking statins. ${ }^{3}$ There is likely to be a higher proportion today, with changes in clinical guidelines and measures to introduce adherence to these. This means that for some patients the benefit of taking a statin was already included before the model applied a further reduction in CVD mortality. Since CVD risk in these people without statins may have been higher, this again would make the benefits of statin therapy more widespread.

Many will argue that quality of life is as important as quantity. The model provides no data on the effect on the length of time lived without disability, yet many people worry about this as much or more so than about death itself. It would be fair say that someone with a lower risk of death from CVD is also at a lower risk of disability from the same cause-such as from symptoms of tiredness and shortness of breath from heart failure or the weakness associated with ischaemic stroke. These benefits would extend beyond those who gain lifespan from statin therapy to those who gain quality of life but not life expectancy, also meaning benefits could be more widespread than suggested by the model results.

Could the findings here be confirmed with data from real people? It seems unlikely. Randomised controlled trials are designed to balance confounding factors in different study populations whereas this type of analysis requires them to be balanced between exactly matched individuals. Trials also follow for a fixed period of time rather than the entire lives of the population studied, and so will struggle to directly measure lifespan gain. Confirmation might be possible by retrospectively matching patients and extrapolating from existing trial databases though this would introduce a very large degree of bias, and even if it were possible would still not give perfect matches. A prospective study to test this would require the randomisation of large numbers of exactly matched individuals to be followed for an extended period of time. Aside from the practical complexities of recruiting such a population, conducting such a study would probably not be ethical.

Although significant, these critiques are unlikely to change the headline conclusions of the study. At most, they would make the numerical results in real life different to those produced in the model. Since the extent of these effects is unknown, it is not feasible to apply error bars to the estimates of lifespan gain.

Broadly accepting the results leads to challenging questions for producing clinical guidelines on the primary prevention of CVD. Those who benefit from statin therapy benefit more if it is started earlier. Therefore it could be argued that focusing on lifetime risk makes more sense than looking at risk over a fixed period of time, as is currently the case. The problem with this is that, as the authors state, the lifetime CVD mortality risk for the average man is $37 \%$. With an average risk level so high, how is one to set a threshold beyond which therapy is appropriate and below which it is not? With this approach, these drugs could be advisable for virtually everyone. To many people this would feel like a step too far in our increasingly medicalised world.

Perhaps a better option is to share these decisions with patients so they can be made in collaboration, depending on the patients' own risk levels and how they weigh these up. Previous work by the same research group has gone some way in quantifying that many patients would not want to take medications for the rest of their lives even for a given gain in life expectancy. ${ }^{4}$ The threshold at which medication becomes worthwhile varies between individuals. The current paper complicates the matter further as it may not be a guarantee of a certain gain in life expectancy that medicine offers. Rather, it is the smaller probability of a larger lifespan gain balanced against a larger probability of no gain. As the authors show in their survey of public views, the two scenarios are valued very differently between people. A $10 \%$ chance of gaining 10 years is not valued in the same way as a $100 \%$ chance of gaining 1 year, despite the mathematics suggesting that they should be equal. Clear decision aids will be needed to help people (and indeed, doctors) understand the statistics as they apply to their own selves and to balance this against their own view of the downsides of taking a medication.

Finally, it is worth considering that the effects demonstrated here may not apply only to the particular scenario of statin therapy but to other medical decisions and even other areas of medicine such as screening for the early detection of cancer. ${ }^{5}$ The study highlights the problem of focusing on disease-specific mortality rather than total mortality in the evidence used to recommend various medical interventions. The two measures are taken as similar but they do not necessarily follow each other as well as researchers would sometimes like to think.

Discussions around the clinical use of statins often generate a wide variety of views, with the drugs portrayed as either being lifesaving or harmful, depending on what you read. ${ }^{6}$ For any changes to existing practice to be useful, a wide discussion is needed, including the medical community and the general public. Further work is needed to apply and explain these results in conversations with individual patients, including reference to the limitations and uncertainty of the data. The authors have generously published the full code of their statistical model with open access so others are able to develop their own findings from the work.

Competing interests None declared. 
Provenance and peer review Commissioned; internally peer reviewed.

Open Access This is an Open Access article distributed in accordance with the Creative Commons Attribution Non Commercial (CC BY-NC 4.0) license, which permits others to distribute, remix, adapt, build upon this work noncommercially, and license their derivative works on different terms, provided the original work is properly cited and the use is non-commercial. See: http:// creativecommons.org/licenses/by-nc/4.0/

\section{REFERENCES}

1. Finegold J, Shun-Shin MJ, Cole GD, et al. The distribution of lifespan gain from primary prevention intervention. Open Heart doi:10.1136/ openhrt-2015-000343. In press.
2. Office for National Statistics. Mortality Statistics: Metadata. Secondary Mortality Statistics: Metadata. 2015. http://www.ons.gov.uk/ons/rel/ vsob1/mortality-statistics-deaths-registered-in-england-and-walesseries-dr-/2012/index.html

3. Fleetcroft R, Schofield P, Ashworth M. Variations in statin prescribing for primary cardiovascular disease prevention: cross-sectional analysis. BMC Health Serv Res 2014;14:414.

4. Fontana M, Asaria P, Moraldo M, et al. Patient-accessible tool for shared decision making in cardiovascular primary prevention balancing longevity benefits against medication disutility. Circulation 2014;129:2539-46.

5. Independent U. K. Panel on Breast Cancer Screening. The benefits and harms of breast cancer screening: an independent review. Lancet 2012;380:1778-86.

6. McCartney M. Statins for all? BMJ 2012;345:e6044. 